\title{
Bronchial Epithelial Cells Promote the Differentiation of Th2 Lymphocytes in Airway Microenvironment through Jagged/Notch-1 Signaling after RSV Infection
}

\author{
Ling Qin ${ }^{a}$ Ke-zi Qiu ${ }^{b}$ Cheng-ping Hu ${ }^{a}$ Guo-jun Wu ${ }^{b}$ Li-li Wang ${ }^{b}$ \\ Yu-rong Tan ${ }^{a, b}$ \\ ${ }^{a}$ Respiratory Department, Xiangya Hospital, Central South University, Changsha, China; \\ ${ }^{\mathrm{b}}$ Department of Basic Medicine, Xiangya School of Medicine, Central South University, Changsha, China
}

\section{Keywords}

Airway hyperresponsiveness · Human bronchial epithelial cells $\cdot$ RSV $\cdot$ T helper cells

\begin{abstract}
Background: The aim of this study was to investigate the role of Notch-1 signaling through Notch-1 ligands on bronchial epithelial cells (BECs) in regulating the development of $T$ helper 2 (Th2) lymphocytes after RSV infection. Methods: Firstly, we analyzed the expression of cytokines and Notch-1 ligands in BECs by using real-time PCR. Then, RSV-infected $\mathrm{BEC}$ s were co-cultured with $\mathrm{CD} 4^{+} \mathrm{T}$ cells in a transwell chamber for $48 \mathrm{~h}$, and differentiation of T cells in the lower chamber was determined using flow cytometry and real-time PCR. JAG1 siRNA was then used to determine the effects of Jagged/Notch-1 signaling on the differentiation of Th2. An RSVinfected mouse model was also used to analyze the secretion of Th differentiation-associated cytokines in serum and lung tissues using ELISA, the histopathological changes using $\mathrm{HE}$ staining, and the expression of JAG1 and JAG2 in BECs. Results: The results showed that RSV promoted the expression of Th2-type cytokines and Jagged-1 and inhibited the expression of Jagged-2 in normal BECs. RSV-infected BECs in-
\end{abstract}

duced Th2 differentiation. In addition, JAG1 downregulation inhibited the differentiation of Th2 and promoted differentiation of Th1. In the RSV-infected mouse model, the RSV titer, inflammation decreased with time. IL-4 and IL-17 increased on day 28 and 60 , while IFN $y$ increased on day 7 and 28 . Moreover, the expression of Jagged-1 increased and that of Jagged-2 decreased in BECs, which was consistent with IL-4 production in lung tissues. Conclusion: Our data showed that BECs had the potential to promote the differentiation of Th2 lymphocytes through Jagged-1/Notch-1 signaling.

(c) 2019 S. Karger AG, Basel

\section{Introduction}

Respiratory syncytial virus (RSV) dramatically increases the risk of airway hyper-responsiveness (AHR) after an acute infection in infants and young children $[1,2]$. A number of studies have demonstrated a relationship between infants with severe RSV infection and

Edited by: A. Haczku, Sacramento, CA, USA.

Ling Qin and Ke-zi Qiu contributed equally to this work.

\section{KARGER}

(c) 2019 S. Karger AG, Basel

E-Mail karger@karger.com

www.karger.com/iaa
Dr. Yu-rong Tan

Respiratory Department, Xiangya Hospital; Department of Basic Medicine

Xiangya School of Medicine, Central South University

Changsha, Hunan 410078 (China)

E-Mail hope7@126.com 
the subsequent development of asthma later in childhood. However, the mechanisms by which RSV may be involved in the development of post-bronchiolitis asthma and allergy are poorly understood. The airway epithelium is the first line of defense against inhaled allergens, viruses, and environmental pollutants through inflammatory, immune, and regenerative responses. It also plays a significant role in initiating pulmonary diseases, particularly in the case of RSV, since this virus replicates and persists only in the respiratory epithelium [3,4]. The long persistence of virus may undermine the physiological homeostasis of airway microenvironment and lead to adaptive remodeling of airway ultra-structure through interaction with host proteins [5]. Our previous study revealed that bronchial epithelial cells (BECs) can act as antigen-presenting cells. In addition, RSV infection in the epithelium promoted the differentiation of T helper 2 (Th2) and Th17 lymphocytes [6-8]. However, the mechanisms remain unclear.

According to previous results, we observed that Notch-1 in the lungs increased after RSV infection (unpublished data). The Notch signaling pathway is a highly evolutionarily conserved cell signaling pathway in most multicellular organisms that regulates proliferation, differentiation, cell fate, progenitor, and stemcell self-renewal in both embryonic and adult tissues. Reports indicate that it is involved in AHR [9]. Several studies have demonstrated that BECs can express all the ligands of Notch-1, which is correlated with the differentiation of Th lymphocytes [10]. In mammals, there are 4 Notch receptors (Notch 1-4) and 5 Notch ligands (Delta1, Delta3, Delta4, Jagged1, and Jagged2), which can differentially affect the Notch-expressing cells [11]. Previous studies that focused on dendritic cells in the lungs revealed that Delta or Jagged ligands expressed on dendritic cells may influence the ability of naive $\mathrm{CD}^{+} \mathrm{T}$ cells to differentiate into Th1 or Th2 lineages [12]. However, it is likely that BECs also have an important role in Th cell differentiation or transdifferentiation, since all ligands of Notch-1 can be detected in the BECs.

It is therefore necessary to understand whether Notch-1, through different ligands on BECs, have distinct roles in regulating Th development. To address this question, we analyzed the expression of Notch-1 ligands in BECs and observed their effects in Th cell differentiation in this study. A mouse model was also established to analyze the correlation of JAG1 and Th differentiation.

\section{Materials and Methods}

\section{Culture and Infection of RSV}

The RSV strain A2 was expanded in Hela cells supplemented with $5 \%$ fetal bovine serum (FBS). The infective process was allowed to proceed until syncytia were observed. The cells were subjected to three successive freeze-thaw cycles followed by re-suspension in fresh medium. The RSV was further purified by centrifugation at $2,500 \mathrm{~g}$ for $10 \mathrm{~min}$ at $4{ }^{\circ} \mathrm{C}$ and filtered through a $0.22-\mu \mathrm{m}$ filter. The virus titer was determined by a plaque assay. $60-70 \%$ of the confluent BECs were infected with RSV at multiplicity of infection (MOI) of 0.1 . The virus was allowed to incubate for $2 \mathrm{~h}$ at $37^{\circ} \mathrm{C}$ in serum-free DMEM. Thereafter, the nonabsorbed virus was removed and washed twice. The cells were then cultured in fresh medium with $2 \% \mathrm{FBS}$ and subcultured as needed. RSV infection was verified by immunofluorescence with a polyclonal rabbit anti-NS1 antibody (Santa Cruz Biotechnology, TX, USA) as a primary antibody and Cy3-conjugated secondary antibody (Santa Cruz Biotechnology, TX, USA).

\section{Separation of $C D 4^{+}$T Cells}

Heparinized blood was obtained from the blood bank in Xiangya Hospital, Central South University. The blood samples were from healthy male adults of approximately similar age and who did not smoke. RSV antibody was negative in the samples. Peripheral blood mononuclear cells (PBMCs) were isolated by Ficoll-Conray density gradient liquid (Haoyang Company, Tianjin, China). Subsequently, $\mathrm{PBMCs}$ were incubated in complete medium at $37^{\circ} \mathrm{C}$ in $5 \% \mathrm{CO}_{2}$ for $2 \mathrm{~h}$ until the monocytes adhered to the bottom of culture flasks. The lymphocytes suspended in the medium were isolated using a $\mathrm{CD}^{+} \mathrm{T}$ Cell Isolation Kit (Miltenyi Biotec, Bergisch Gladbach, Germany) by positive selection of $\mathrm{CD}_{4} 5 \mathrm{RA}^{+} \mathrm{CD} 4^{+} \mathrm{T}$ cells and negative selection of $\mathrm{CD} 45 \mathrm{RO}^{+} \mathrm{CD} 4^{+} \mathrm{T}$ cells. Cells were cultivated at $37^{\circ} \mathrm{C}$ in $10 \%$ human $\mathrm{AB}$ plasma-containing RPMI 1640 medium, supplemented with penicillin-streptomycin. A total

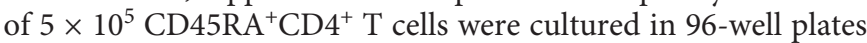
along with $1 \times 10^{5}$ beads coated with $5 \mu \mathrm{g} / \mathrm{mL}$ of anti-CD3 and anti-CD28, according to the manufacturer's instructions.

\section{Co-Culture of $C D 4^{+} T$ Cells with RSV-Infected BECs}

Transwell chambers with 24-well inserts were used in the coculture assay. RSV-infected BECs ( $10^{5}$ cells) with or without JAG1 RNAi were plated in the top chamber and incubated for $24 \mathrm{~h}$. CD $4^{+}$ $\mathrm{T}$ cells were placed in the lower chamber at a 1:1 ratio with BECs. After $48 \mathrm{~h}$ of incubation, lymphocytes were collected and assayed by flow cytometry.

\section{Flow Cytometry Analysis}

To detect the Th cell differentiation, the ratio of $\mathrm{CD} 4^{+} \mathrm{IFN} \gamma^{+}$ cell (Th1), CD4 $4^{+} \mathrm{IL}-4^{+}$cell (Th2), CD $4^{+} \mathrm{IL}-17^{+}$cell (Th17), and CD $25^{+}$Foxp $^{+}$cell (Treg cells) were analyzed by flow cytometry analysis. Lymphocytes were then pretreated with $2 \mathrm{~mL}$ of monen$\sin$ (BioLegend, CA, USA) for $6 \mathrm{~h}$ and then incubated for $30 \mathrm{~min}$ at $4{ }^{\circ} \mathrm{C}$ with FITC-anti-CD4, PE-conjugated anti-IFN $\gamma$, IL-4, IL17, Alexa Fluor 647 anti-Foxp3, and PE-anti-CD25 mAbs (BD Biosciences, CA, USA). The cells were subsequently washed twice and resuspended in PBS containing 1\% FBS and 0.1\% NaN3 (SigmaAldrich, Beijing, China) and immediately analyzed with FACS (Becton Dickinson, NY, USA). Isotype-matched antibodies were used as controls.
Qin/Qiu/Hu/Wu/Wang/Tan 


\section{Real-Time PCR}

The five Notch-1 ligands including Jagged-1, Jagged-2, DLL1, DLL3, and DLL4 on BECs and transcription factors of Th differentiation including T-bet (Th1), GATA-3 (Th2), ROR $\lambda \tau$ (Th17), and Foxp3 (Treg cells) in Th cells were assayed by real-time PCR. Following the manufacturer's guidelines, RNA was isolated from BECs in the top chamber or CD4 ${ }^{+} \mathrm{T}$ cells in the lower chamber using TRIZOL ${ }^{\circledR}$ Reagent (Invitrogen, CA, USA). Afterwards, the RNA was incubated at $37^{\circ} \mathrm{C}$ for $30 \mathrm{~min}$ in DNase I solution to remove the contaminating DNA. The RNA was subsequently treated using RNeasy ${ }^{\circledR}$ MinElute $^{\mathrm{TM}}$ Cleanup Kit (Qiagen, MA, USA). RNA yield and quality were assayed by detecting the ratio of A260 to A280 values and denaturing agarose gel electrophoresis. After the first strand cDNA synthesis using SuperScript III Reverse Transcriptase, real-time PCR was performed by SYBR Green Real-Time PCR Kit (Takara, Beijing, China) at $95^{\circ} \mathrm{C}$ for $10 \mathrm{~min}$, then at $95^{\circ} \mathrm{C}$ for $15 \mathrm{~s}$, and at $60^{\circ} \mathrm{C}$ for $1 \mathrm{~min}$ for 35 amplification cycles. The $\Delta \mathrm{Ct}$ (average $\mathrm{Ct}-$ average $\mathrm{Ct}$ of Homo18SrRNA) and $\Delta \Delta \mathrm{Ct}[\Delta \Delta \mathrm{Ct}=\Delta \mathrm{Ct}$ (experimental) $-\Delta \mathrm{Ct}$ (control) $]$ were calculated. The relative expression was calculated from experiment to control as $2^{-\Delta \Delta \mathrm{Ct}}$ [13]. The following primers were used in the assays: Jagged-1: 5-GCTACTACTGCGACTGTCTTCCCG-3 and 5-AGATACAGCGATAACCATTAACCAAA-3 (121 bp); Jagged-2: 5-CGCCCGTAACAACCGTCTC-3 and CAATAACTGTGCCCGCCTCAC (330 bp); DLL1: 5-GGACTCGGGCTGTTCAACTTC-3 and AGTCTTGCCATCTCACTTCCATTT (118 bp); DLL3: 5-CAGGTGCGGATGGAGGAGAD-3 and GGGCTTGAGGAGGTCACGAT (150 bp); DLL4: 5-GACAGCCCGAAAGACAGATAGG-3 and 5-GTGGGTCAGAACTGGTTATTGGA-3 (229 bp); T-bet: 5-ACTGCTCGAAACTCAGCCTCAT-3 and 5-ACCCAGTTCATTGCCGTGAC-3 (316 bp); GATA-3, 5-GCTTTGAACAAATGATTCGCCTA-3 and 5-AGGATGCCAAGAAGTTTAAGGAATAT-3 (203 bp); ROR $\lambda \tau: 5-T C T T C T C T C A A G T A T T G G C A G G T T T-3$ and 5-TCTTTCCCTACTGTTCGTTCACCA-3 (119 bp); Foxp3, 5-GTGTTGAGTGAGGGACAGGATTG-3 and 5-AAGGAAAGGAGGATGGACGAAC-3 (381 bp).

\section{RNAi}

JAG1 siRNA and control siRNA were purchased from Thermofisher (Shanghai, China). Cells were seeded into 6-well plates at $10^{6}$ cells per well. $90 \%$ of the confluent monolayer cultures were incubated with a final concentration of $10 \mathrm{nM}$, siRNA, or negative control using Lipofectamine 2000 for $24 \mathrm{~h}$. Subsequently, the medium was replaced by a fresh medium and co-cultured with $\mathrm{CD} 4^{+}$ $\mathrm{T}$ cells for $48 \mathrm{~h}$.

\section{Mouse Models}

One-week-old male C57 mice weighing 8-10 g from the same mother were obtained from the experimental animal center, Central South University, and maintained in a pathogen-free environment. The mice were randomly divided into control group $(n=20)$ and RSV group $(n=20)$ by random number table method. All the procedures were reviewed and approved by the Institutional Animal Care and Use Committee. The virus titer in the inoculum was determined by plaque assay on the same day as the inoculation of mice. Mice were made to inhale $5 \%$ halothane before $50 \mu \mathrm{L}$ of PBS containing $10^{6} \mathrm{pfu}$ of RSV was intranasally inoculated. Control mice received placebo inoculums. The mice were studied on day 7,28 , and 60 with 6 mice from each group after being anesthetized with a lethal dose of chloral hydrate. RSV infection was assayed by real-time PCR and immunohistochemistry.

RSV-Infected BECs Promoted Th2

Differentiation

\section{Immunohistochemistry}

Mice were perfused with PBS after sacrifice, followed by perfusion fixation with $4 \%$ paraformaldehyde diluted in $0.1 \mathrm{M}$ PBS for $4 \mathrm{~h}$. The left lung was then removed, rinsed with $0.9 \%$ sodium chloride solution, and fixed in $10 \%$ formaldehyde solution for $24 \mathrm{~h}$ ahead of paraffin sectioning. Following routine deparaffin, hydration, tissue antigen retrieval, endogenous peroxidase inactivation, and nonspecific staining blockage, rabbit anti-JAG1 or JAG2 polyclonal antibody (Santa Cruz Biotechnology, TX, USA) was added and incubated overnight at $4{ }^{\circ} \mathrm{C}$. After thorough rinsing, the slides were incubated with biotinylated universal secondary antibody and then horseradish peroxidase-labelled streptomycin-avidin in turn. The slides were colored with DAB staining solution for 15 min and then observed under a high-power microscope. The integral optical density values of JAG1 and JAG2 protein in BECs were measured by CMIAS- 8 color pathological image analysis system.

\section{Western Blot Analysis}

Lysates of BECs or lung tissues ( $75 \mu \mathrm{g}$ ) were quantified by A280 (Eppendorf, Hamburg, Germany) and separated on 10\% SDSPAGE and then transferred to polyvinylidene fluoride membrane (Millipore, MA, USA). Membranes were blocked with 5\% skim milk and incubated with rabbit anti-human Notch-1 and CSL (Cell Signaling Technology Inc., MA, USA) at $4{ }^{\circ} \mathrm{C}$ for the entire night. After being washed, membranes were incubated with horseradish peroxidase-conjugated goat anti-rabbit IgG (1:4,000; ProteinTech Group Inc., IL, USA) for $1 \mathrm{~h}$ at room temperature. Antibody-antigen complexes were then detected using an ECL chemiluminescent detection system (Gene Co., Ltd., Hong Kong, China). GAPDH was used as a loading control. A densitometry analysis was performed using Alpha-Ease software version 2200.

\section{Cytokine Measurement}

The eyeball blood was extracted from mice, left to stand for 20 $\mathrm{min}$, and then centrifuged at $400 \mathrm{~g} / \mathrm{min}$ for $15 \mathrm{~min}$ to obtain serum. The total protein from lung tissues was extracted according to the instructions. Mouse IFN $\gamma$, IL-4, and IL-17 concentrations in serum and lung tissues were determined using ELISA kits from R\&D Systems (USA).

\section{Statistical Analysis}

Statistical analysis was done using SPSS software 16. All values were expressed as mean \pm SE. Means were compared by the use of one-way analysis of variance (ANOVA) and Bonferroni post-hoc analysis between two groups. A $p$ value $<0.05$ was considered significant.

\section{Results}

\section{RSV Promoted Th2-Type Cytokines Secretion from}

BECs

RSV infection was confirmed by observing cytopathic effects and indirect immunofluorescence at 24 and $48 \mathrm{~h}$ after infection (MOI =0.1). Red fluorescence was noted within the cells (Fig. 1a). Previous reports [14-17] showed thymic stromal lymphopoietin (TSLP), granulocyte-

Int Arch Allergy Immunol 2019;179:43-52 


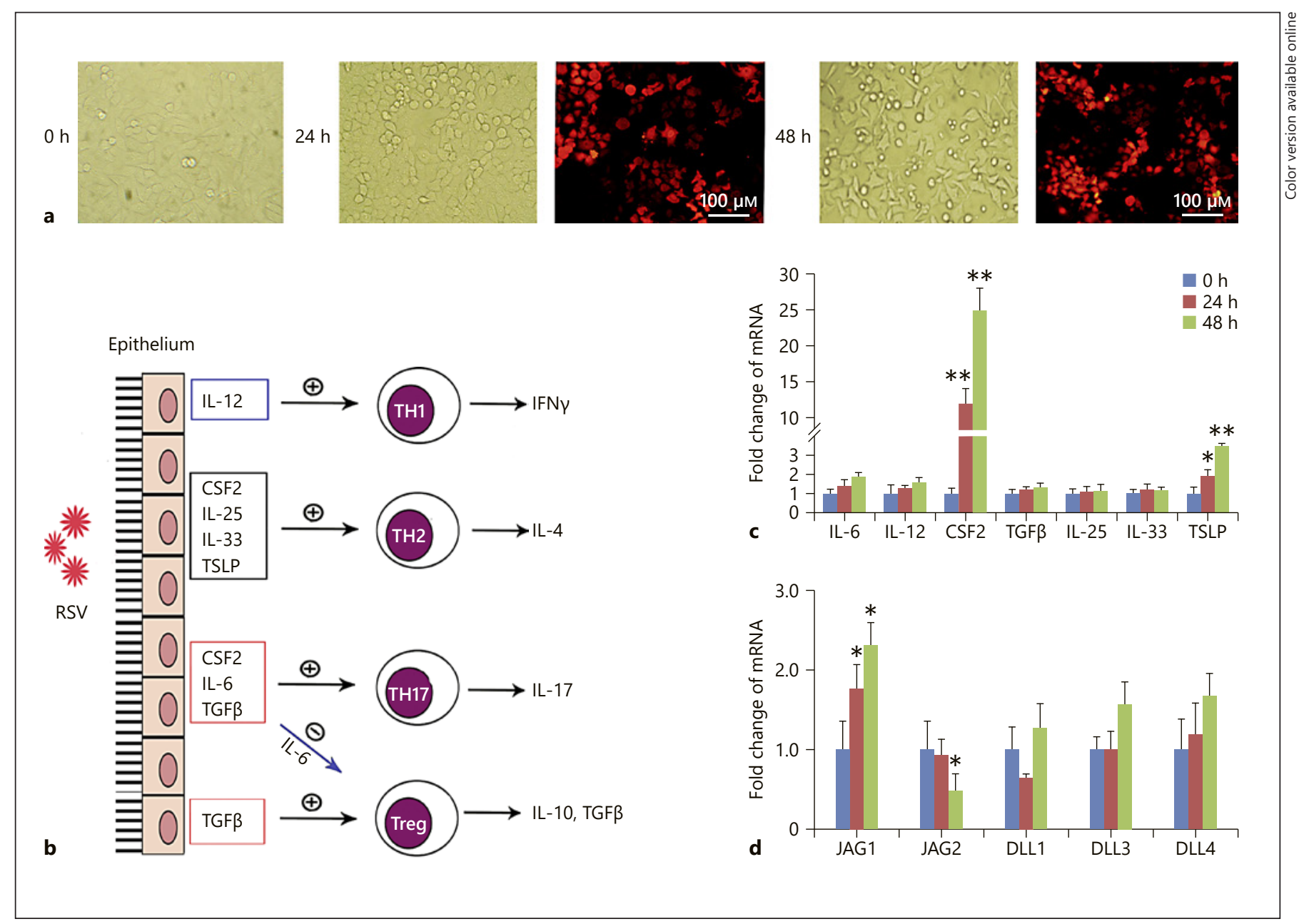

Fig. 1. Effects of RSV on the expression of cytokines and Notch-1 ligands in BECs. a RSV infection was assayed by indirect immunofluorescence. $\mathbf{b}$ Effects of epithelium-derived cytokines on the differentiation of Th. c The expression levels of cytokines from
BECs were determined by real-time PCR. $\mathbf{d}$ The expression levels of Notch-1 ligands were determined by real-time PCR. Results are mean values \pm SD of 6 independent experiments. ${ }^{*} p<0.05$ and ** $p<0.01$ vs. zero. Zero represents normal control. macrophage colony stimulating factor (CSF2), IL-25, and IL-33 produced by epithelial cells were crucial for Th2 differentiation. Epithelium-derived CSF2, IL-6, and TGF $\beta$ promote Th17 differentiation mainly through activation dendritic cells. TGF $\beta$ promotes Treg differentiation, but IL-6 inhibits Treg differentiation. IL-12 promotes Th1 differentiation (Fig. 1b). Therefore, the above cytokine expressions from RSV-infected BECs were assayed by real-time PCR. The results showed that RSV promoted the expression of CSF2 and TSLP (Fig. 1c).

The expression of the five ligands of Notch-1 in BECs was determined. Results of real-time PCR showed that RSV promoted the expression of JAG1 at 24 and $48 \mathrm{~h}$ but inhibited the expression of Jagged 2 at 48 h (Fig. 1d).

\section{RSV Promoted Th2 Differentiation through JAG1/}

Notch-1 Signaling

BECs, with or without RSV infection, were cultured and subsequently co-cultured with $\mathrm{CD} 4^{+} \mathrm{T}$ cells. The results of flow cytometry analysis showed that RSV promoted the differentiation of Th2 cells (Fig. 2a). Furthermore, the mRNA expression of GATA-3 also increased significantly after RSV infection (Fig. 2b).

To understand the effects of JAG1 on the differentiation of Th cells, the BECs were cultured for $48 \mathrm{~h}$ after pretreatment with JAG1 RNAi or control RNAi. The inhibitory effects of the RNAi to JAG1 were verified by realtime PCR (Fig. 3a). The BECs were then infected with RSV for $24 \mathrm{~h}$ and co-cultured with $\mathrm{CD}^{+} \mathrm{T}$ cells for an 

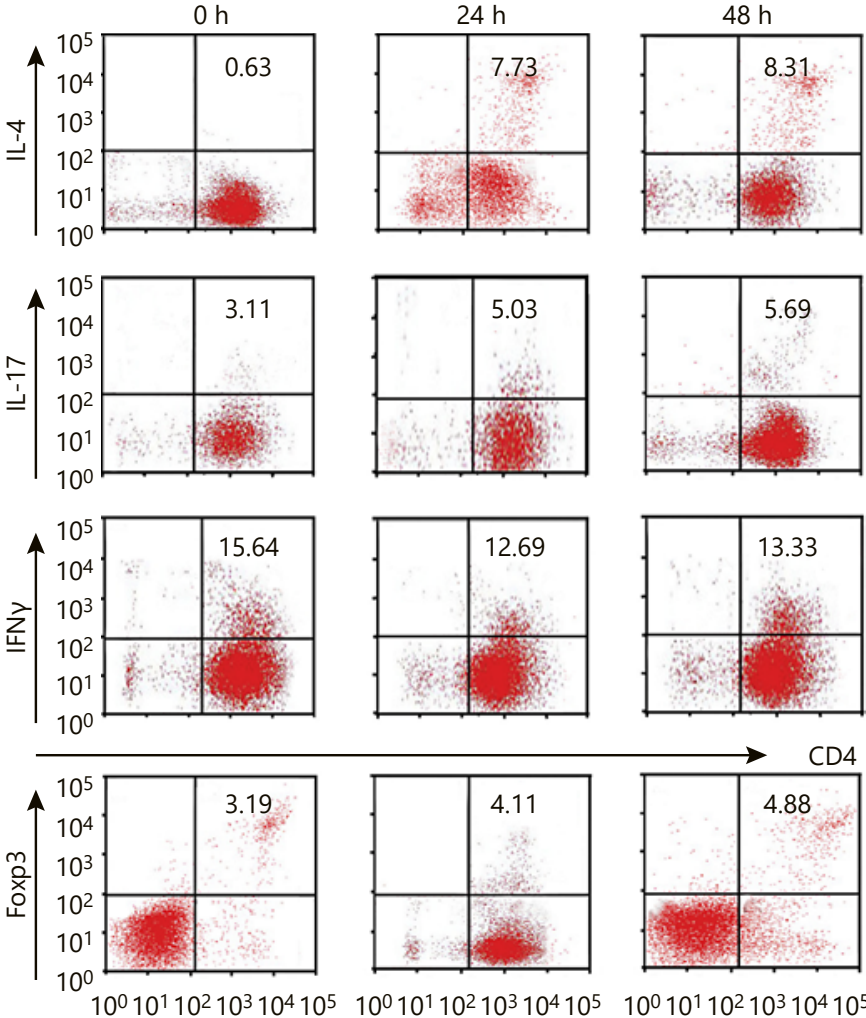

$10^{0} 10^{1} 10^{2} 10^{3} 10^{4} 10^{5}$
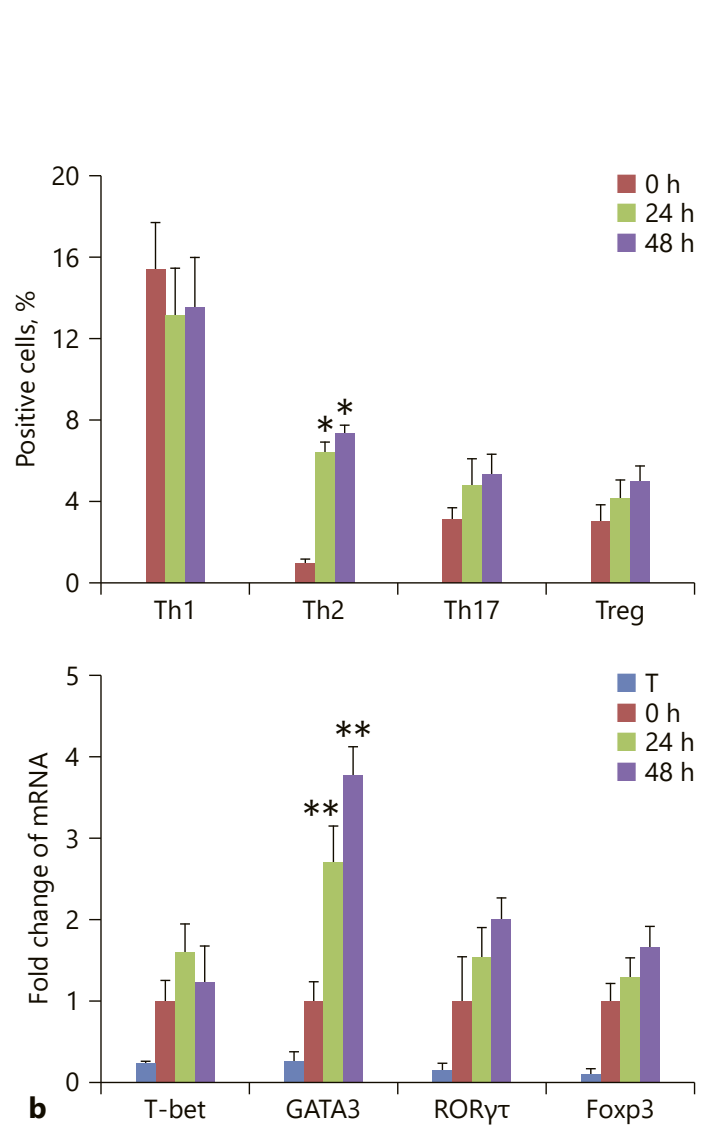

Fig. 2. Differentiation of Th cells after co-culture with BECs was assayed by flow cytometry (a) and real-time PCR (b). RSV-infected BECs promoted the differentiation of Th2 cells. The results were from 6 independent experiments. ${ }^{*} p<0.05$ and ${ }^{* *} p<0.01$ vs. zero. Zero represents normal control; T represents Th cells without coculture with BECs. additional $48 \mathrm{~h}$. Using real-time PCR, the study revealed that JAG1 RNAi inhibited the expression of GATA3 mRNA (Th2 cells) and promoted the expression of T-bet mRNA (Th1 cells) (Fig. 3b).

Activation of Notch-1 in Th cells was detected and found to decrease significantly after co-culture with JAG1 RNAitreated BECs when compared with that of the control group $(p<0.05$, Fig. $3 c)$. However, the DNA-binding protein CSL of Notch-1 also decreased significantly after co-culture with JAG1 RNAi-treated BECs ( $p<0.05$, Fig. 3d).

\section{RSV Infection Significantly Induced Airway \\ Pathological Changes}

The expression of RSV in lung tissue was very strong on the 7th day of RSV infection, although the expression gradually decreased with time. On the 60th day after infection, the expression of RSV in lung tissue was the lowest

RSV-Infected BECs Promoted Th2 Differentiation
(Fig. 4a). The pathological changes in the lung included significant epithelial denudation and inflammatory infiltration which were noted to decrease with time in the RSV group (Fig. 4b). The influence of RSV on the cytokines in lung tissues was found to be greater than in serum. It was discovered that the three cytokines in the RSV group were not significantly different from those in the control group in serum (Fig. 4c). However, in the lung tissues, IL-4 increased significantly on the 28th and 60th day after infection, IL-17 was significantly higher on the 28th and 60th day after infection, while IFN $\gamma$ was significantly higher on the 7 th and 28th day after infection $(p<0.05)$ (Fig. $4 d$ ).

\section{Expression of JAG1 and JAG2 in Lung Tissues}

The expression levels of JAG1 and JAG2 in BECs were examined using immunohistochemical staining 7,28, and 60 days after infection. Although JAG1 expression de- 

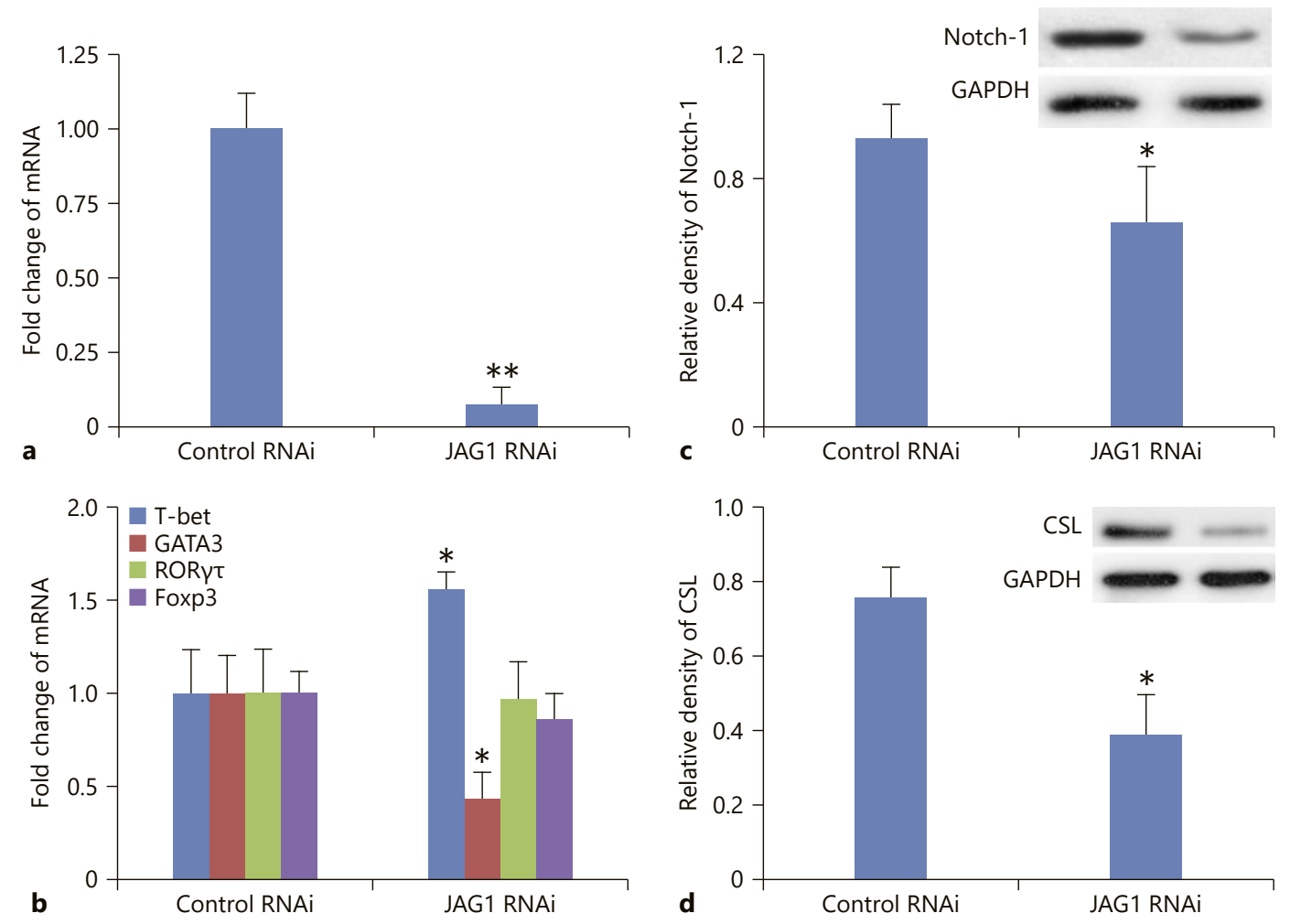

Fig. 3. The effects of JAG1 RNAi on the differentiation of Th cells and the activation of Notch-1. a BECs were incubated with a final concentration of $10 \mathrm{nM}$, siRNA, or control RNAi using Lipofectamine 2000 for $24 \mathrm{~h}$. The inhibitory effects of RNAi on JAG1 were verified by real-time PCR. b Subsequently, the JAG1 RNAitreated BECs were co-cultured with $\mathrm{CD}^{+} \mathrm{T}$ cells for $48 \mathrm{~h}$. The ef- fects of JAG1 RNAi on the differentiation of Th cells were assayed by real-time PCR. The expression of Notch-1 (c) and CSL (d) in $\mathrm{CD} 4^{+} \mathrm{T}$ cells was assayed by Western blot. The results were from 6 independent experiments. ${ }^{*} p<0.05$ and ${ }^{* *} p<0.01$ vs. control RNAi.

mokines. Chronic and repetitive exposure of BECs to stimuli led to airway remolding and AHR. The development of AHR is one of the defining features of asthma. It is associated with dysfunction of BECs. A large number of clinical and basic studies have verified that RSV, which replicates and persists only in the epithelium, is an important risk factor for subsequent AHR.

Previous reports have shown that epithelium-derived CSF2, TSLP, IL-25, and IL-33 are crucial for Th2 differentiation [14]. Epithelium-derived CSF2, IL-6, and TGF $\beta$ promote Th17 differentiation mainly through activation of dendritic cells [15]. TGF $\beta$ promotes Treg differentiation, but IL-6 inhibits Treg differentiation [16]. IL-12 promotes Th1 differentiation [17]. The lung epithelium of asthmatic patients contains a higher number of $\mathrm{TSLP}^{+}$cells. More-
48

Int Arch Allergy Immunol 2019;179:43-52 DOI: 10.1159/000495581
Qin/Qiu/Hu/Wu/Wang/Tan 


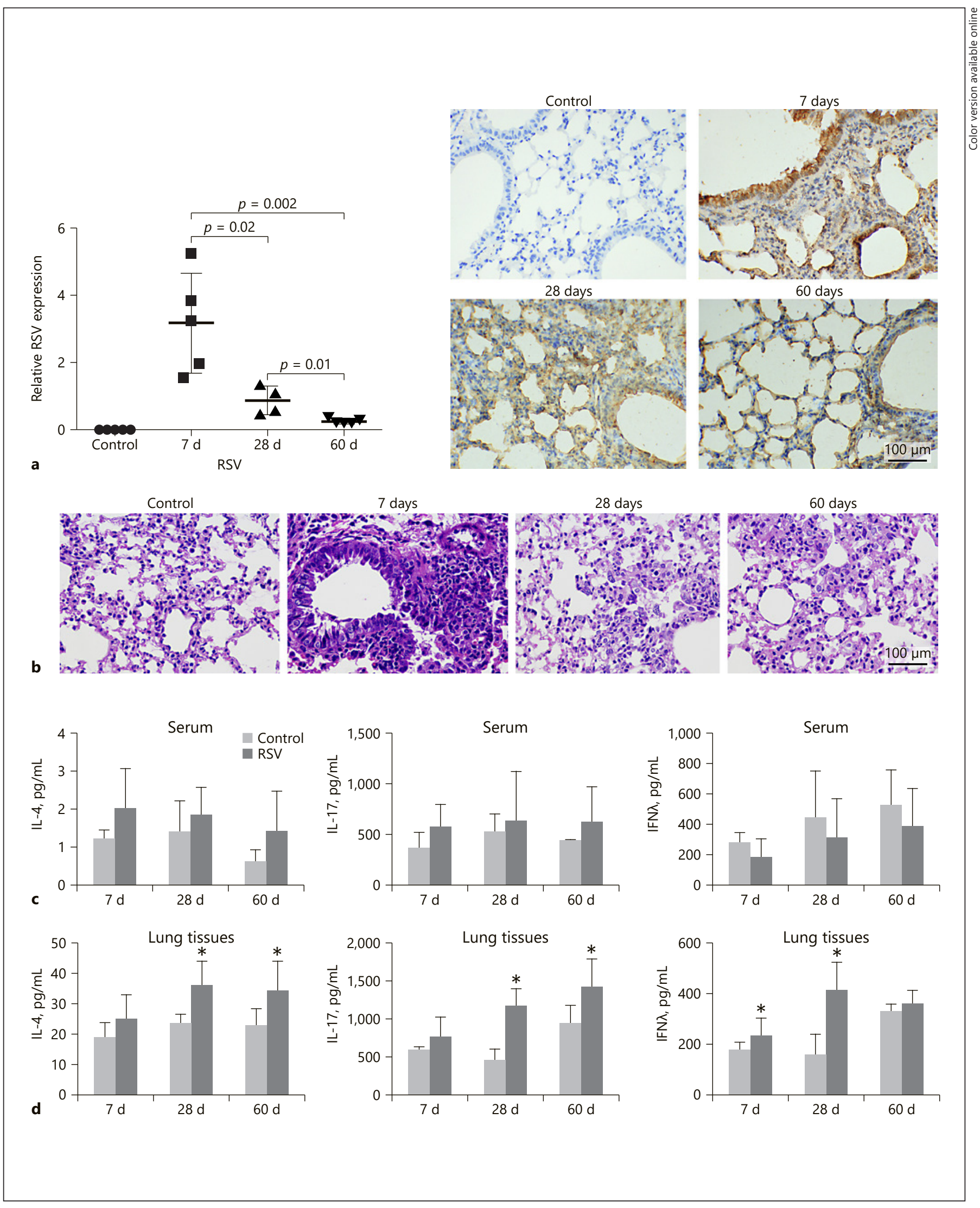

(For legend see next page.)

RSV-Infected BECs Promoted Th2 Differentiation
Int Arch Allergy Immunol 2019;179:43-52 DOI: 10.1159/000495581 


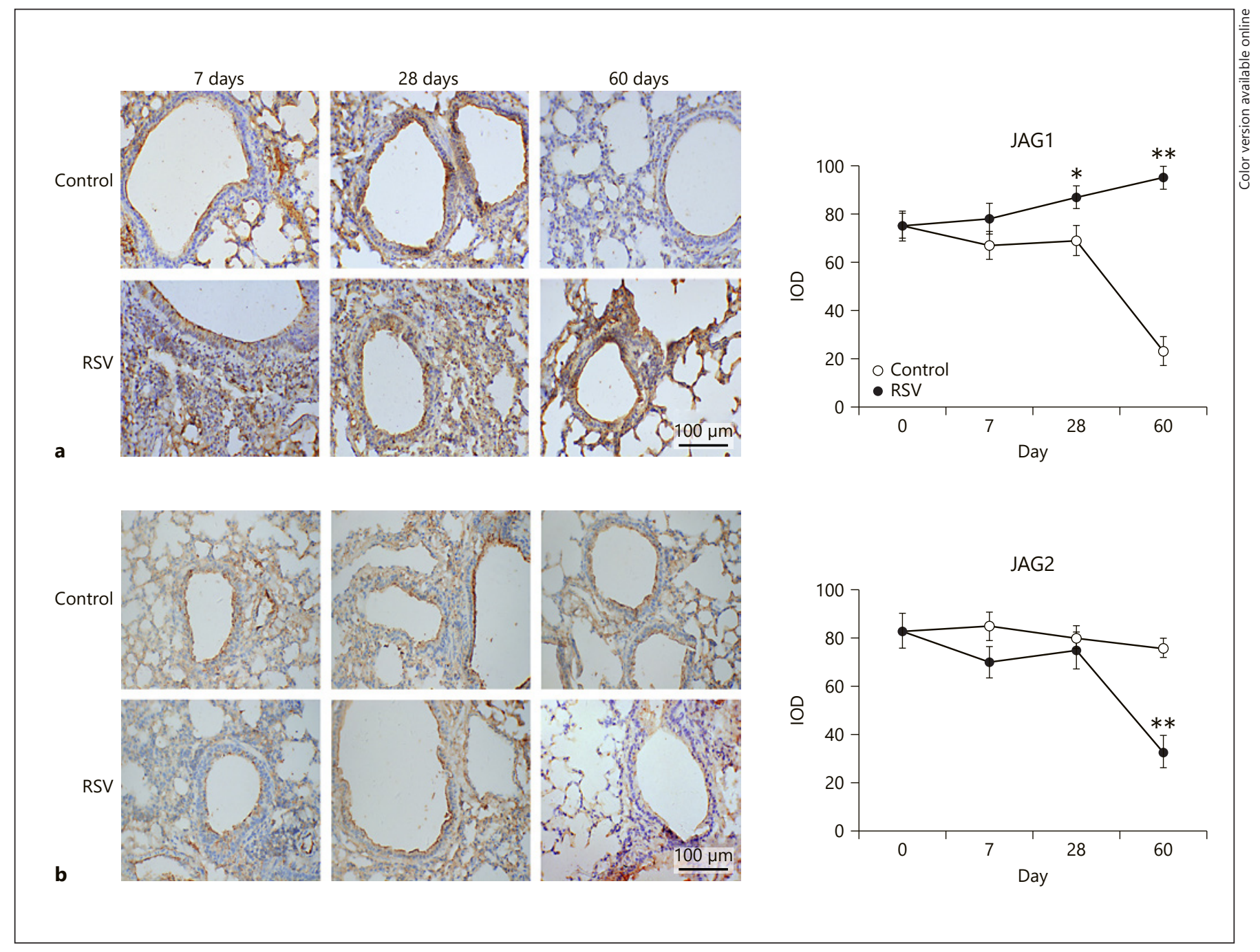

Fig. 5. The expression levels of JAG1 (a) and JAG2 (b) in BECs were assayed by immunohistochemistry. The integral optical density (IOD) values of JAG1 and JAG2 protein in BECs were mea- sured by CMIAS- 8 color pathological image analysis system. The results were from 6 mice. ${ }^{*} p<0.05$ and ${ }^{* *} p<0.01$ vs. control.
Fig. 4. RSV infection significantly induced airway pathological changes. Mice weighing 8-10 $\mathrm{g}$ from the same mother were randomly divided into control and RSV group and made to inhale 5\% halothane before $50 \mu \mathrm{L}$ of PBS containing $10^{6}$ pfu of RSV was intranasally inoculated. Control mice received placebo inoculums. The mice were studied on day 7, 28, and 60 with 6 mice from each group. a RSV infection on day 7, 28, and 60 was assayed by real-time PCR and immunohistochemistry. $\mathbf{b}$ The pathological changes in the lung were examined by HE staining. $\mathbf{c}$ The production of cytokines in serum was assayed by ELISA. d The production of cytokines in lung tissues was assayed by ELISA. IL-4 increased significantly on the 28th and 60th day; IL-17 was significantly higher on the 28th and 60th day, while IFN $\gamma$ was significantly higher on the 7 th and 28 th day after infection in the lung tissues. ${ }^{*} p<0.05$ vs. control. over, the bronchoalveolar lavage samples of these patients have higher concentrations of TSLP protein compared to those of healthy individuals [18]. Higher concentration of TSLP mRNA expression in the lung epithelium is crucial for development of allergic diseases such as asthma [19]. A polymorphism has been identified in the promoter of IL33R which predisposes to a greater risk of developing asthma [20]. IL-25 in epithelial cells is a derived cytokine in Th2-high corticosteroid-responsive asthma [21]. In the current study, we found that CSF2 and TSLP increased significantly. The other cytokines showed no changes $48 \mathrm{~h}$ after RSV infection, indicating RSV-infected BECs have the potential to drive the differentiation of Th2 cells. 
The epithelium also plays a critical role in the development and function of the T cells. Five membrane-bound ligands of Notch-1 are commonly expressed on BECs and are closely associated with the development of Th cells [22]. The present study reveals that BECs can express all five ligands of Notch-1. In addition, RSV promoted the expression of JAG1 and inhibited the expression of JAG2 in BECs $48 \mathrm{~h}$ after RSV infection. Further, JAG1 silencing inhibited the differentiation of Th2 cells and promoted the differentiation of Th1 cells. Consistent with the previous reports, JAG1 promoted Th2 differentiation on dendritic cells [23]. The Th1/Th2 balance plays an important role in the AHR. Th2 inhibits the differentiation of Th1. When Th2 is suppressed, Th1 begins to express itself in large quantities. So, we posit that the increased differentiation of Th1 cells may be a feedback mechanism due to decreased differentiation of Th2 cells. Previous reports also show that JAG2 inhibits the differentiation of $B$ cells [24], indicating that decreased JAG2 promoted the differentiation of B cells and subsequent antibody production.

A mouse model was also used to analyze the correlation of JAG1 and Th differentiation. During acute infection, infants may have extensive and prolonged epithelial damage and denudation. However, on the mouse model after RSV infection, we found epithelial denudation and inflammatory infiltration after 7 days and no significant morphological changes on day 28 and 60. There were no significant changes in cytokines in serum. However, in the lung tissues, on day 7 and 28, the main differentiation was Th1 and, on day 28 and 60, Th2 and Th17. Further, we verified that increased JAG1 and decreased JAG2 were found in BECs. Studies on animal models and humans have shown that simple RSV infection induces Th1 response [25], which may be related to the increase of Th1 on 7 and 28 days after RSV infection. Compared with the control group, JAG1 increased significantly on 28 and 60 days after RSV infection, which was consistent with the expression of IL-4 in the lung and may be related to the imbalance of Th1/Th2 and the induction of Th2 differentiation in the later stage. The increased expression of IL-17 on 28 and 60 days after RSV infection may be related to Th17 differentiation, which may be related to the activation of dendritic cells in the lungs. These indicate that the JAG1 on BECs is a strong factor that drives Th2 differentiation. In addition, we found RSV maintained JAG1 expression, while in control animals it was highly diminished after 60 days, indicating that JAG1 expression was closely related to the age of mice.

There are two potential limitations to the current study. First, in vivo experiments are more complex because various antigen-presenting cells can express
Notch-1 ligands, especially the dendritic cells. Therefore, the effects of Notch-1 ligands on differentiation of Th lymphocytes should be further studied through additional knock-out model for Notch-1 ligands. Second, many scholars believe that the BECs are not the main antigenpresenting cells and that naïve $\mathrm{T}$ cells commonly do not reach the BECs. However, BECs are some of the important effectors and are often in contact with various lymphocytes, indicating that BECs may be important in regulation of Th differentiation or transdifferentiation. Hence, comprehensive studies should be conducted to elucidate the role played by epithelial cells and other antigen-presenting cells in T lymphocyte differentiation.

In summary, activation of JAG1 in BECs promoted the differentiation of Th2 cells, which may be associated with the pathogenesis of RSV causing AHR.

\section{Statement of Ethics}

The study was approved by the Institutional Ethics Committee of Central South University.

\section{Disclosure Statement}

The authors declare no competing interests.

\section{Funding Sources}

This work was supported by Grant 31670121 and 31771277 from the National Natural Science Foundation of China, Grant 2015JJ370 from the Natural Science Foundation of Hunan Province, and 14K108 from the Open Innovation Platform Foundation of Hunan universities.

References

Int Arch Allergy Immunol 2019;179:43-52

1 Backman K, Piippo-Savolainen E, Ollikainen $\mathrm{H}$, Koskela H, Korppi M. Adults face increased asthma risk after infant RSV bronchiolitis and reduced respiratory health-related quality of life after RSV pneumonia. Acta Paediatr. 2014 Aug; 103(8):850-5.

2 Zomer-Kooijker K, van der Ent CK, Ermers MJ, Uiterwaal CS, Rovers MM, Bont LJ; RSV Corticosteroid Study Group. Increased risk of wheeze and decreased lung function after respiratory syncytial virus infection. PLoS One. 2014 Jan;9(1):e87162.

3 Tan YR, Yang T, Liu SP, Xiang Y, Qu F, Liu $\mathrm{HJ}$, et al. Pulmonary peptidergic innervation remodeling and development of airway hyperresponsiveness induced by RSV persistent infection. Peptides. 2008 Jan;29(1):47-56. 
4 Liu X, Qin X, Xiang Y, Liu H, Gao G, Qin L, et al. Progressive changes in inflammatory and matrix adherence of bronchial epithelial cells with persistent respiratory syncytial virus (RSV) infection (progressive changes in RSV infection). Int J Mol Sci. 2013 Sep 3; 14(9):18024-40.

5 Tan YR, Peng D, Chen CM, Qin XQ. Nonstructural protein-1 of respiratory syncytial virus regulates HOX gene expression through interacting with histone. Mol Biol Rep. 2013 Jan;40(1):675-9.

6 Xiang Z, Liang Z, Yanfeng H, Leitao K. Persistence of RSV promotes proliferation and epithelial-mesenchymal transition of bronchial epithelial cells through Nodal signaling. J Med Microbiol. 2017 Oct;66(10):1499-505.

7 Qin L, Tan YR, Hu CP, Liu XA, He RX. Leptin is oversecreted by respiratory syncytial virusinfected bronchial epithelial cells and regulates Th2 and Th17 cell differentiation. Int Arch Allergy Immunol. 2015;167(1):65-71.

8 Liu H, Peng L, Liu C, Tan Y, Xiang Y, Qu X, et al. Activation of Bombesin Receptor Subtype-3 Promotes Antigen-Presenting Action in Human Bronchial Epithelial Cells. Int Arch Allergy Immunol. 2018;175(1-2):53-60.

$9 \mathrm{Hu}$ C, Li Z, Feng J, Tang Y, Qin L, Hu X, et al. Glucocorticoids Modulate Th1 and Th2 Responses in Asthmatic Mouse Models by Inhibition of Notch1 Signaling. Int Arch Allergy Immunol. 2018;175(1-2):44-52.

10 Beck RC, Padival M, Yeh D, Ralston J, Cooke KR, Lowe JB. The Notch ligands Jagged2, Delta1, and Delta4 induce differentiation and expansion of functional human NK cells from CD34+ cord blood hematopoietic progenitor cells. Biol Blood Marrow Transplant. 2009 Sep;15(9):1026-37.
11 Heijink IH, Postma DS, Noordhoek JA, Broekema M, Kapus A. House dust mite-promoted epithelial-to-mesenchymal transition in human bronchial epithelium. Am J Respir Cell Mol Biol. 2010 Jan;42(1):69-79.

12 Liotta F, Frosali F, Querci V, Mantei A, Filì L, Maggi L, et al. Human immature myeloid dendritic cells trigger a $\mathrm{TH} 2$-polarizing program via Jagged-1/Notch interaction. J Allergy Clin Immunol. 2008 Apr;121(4):1000-5.e8.

13 Li L, Liu M, Kang L, Li Y, Dai Z, Wang B, et al. HHEX: A Crosstalker between HCMV Infection and Proliferation of VSMCs. Front Cell Infect Microbiol. 2016 Nov;6:169.

14 Licona-Limón P, Kim LK, Palm NW, Flavell RA. TH2, allergy and group 2 innate lymphoid cells. Nat Immunol. 2013 Jun;14(6):536-42.

15 Ijaz T, Tilton RG, Brasier AR. Cytokine amplification and macrophage effector functions in aortic inflammation and abdominal aortic aneurysm formation. J Thorac Dis. 2016 Aug; 8(8):E746-54.

16 Liao H, Peng X, Gan L, Feng J, Gao Y, Yang S, et al. Protective Regulatory T Cell Immune Response Induced by Intranasal Immunization With the Live-Attenuated Pneumococcal Vaccine SPY1 via the Transforming Growth Factor- $\beta 1-S m a d 2 / 3$ Pathway. Front Immunol. 2018 Aug;9:1754.

17 Regoli M, Man A, Gicheva N, Dumont A, Ivory K, Pacini A, et al. Morphological and functional characterization of IL-12R $\beta 2$ chain on intestinal epithelial cells: implications for lo$\mathrm{cal}$ and systemic Immunoregulation. Front Immunol. 2018 May;9:1177.

18 Bleck B, Kazeros A, Bakal K, Garcia-Medina L, Adams A, Liu M, et al. Coexpression of type 2 immune targets in sputum-derived epithelial and dendritic cells from asthmatic subjects. J Allergy Clin Immunol. 2015 Sep; 136(3):619-627.e5.
19 Zhou B, Comeau MR, De Smedt T, Liggitt HD, Dahl ME, Lewis DB, et al. Thymic stromal lymphopoietin as a key initiator of allergic airway inflammation in mice. Nat Immunol. 2005 Oct; 6(10):1047-53.

20 Harada M, Hirota T, Jodo AI, Hitomi Y, Sakashita M, Tsunoda T, et al. Thymic stromal lymphopoietin gene promoter polymorphisms are associated with susceptibility to bronchial asthma. Am J Respir Cell Mol Biol. 2011 Jun;44(6):787-93.

21 Cheng D, Xue Z, Yi L, Shi H, Zhang K, Huo $\mathrm{X}$, et al. Epithelial interleukin-25 is a key mediator in Th2-high, corticosteroid-responsive asthma. Am J Respir Crit Care Med. 2014 Sep; 190(6):639-48

$22 \mathrm{Xu}$ LL, Fu HX, Zhang JM, Feng FE, Wang $\mathrm{QM}$, Zhu XL, et al. Impaired function of bone marrow mesenchymal stem cells from immune thrombocytopenia patients in inducing regulatory dendritic dell differentiation through the Notch-1/Jagged-1 signaling pathway. Stem Cells Dev. 2017 Nov 15;26(22): 1648-61.

23 Ronzani C, Casset A, Pons F. Exposure to multi-walled carbon nanotubes results in aggravation of airway inflammation and remodeling and in increased production of epithelium-derived innate cytokines in a mouse model of asthma. Arch Toxicol. 2014 Feb; 88(2):489-99.

24 Amsen D, Blander JM, Lee GR, Tanigaki K, Honjo T, Flavell RA. Instruction of distinct CD4 T helper cell fates by different notch ligands on antigen-presenting cells. Cell. 2004 May;117(4):515-26.

25 Santini F. Human respiratory syncytial virus and Th1 chemokines. Clin Ter. 2015;166(3): e203-8. 